\title{
Composição Mineral das Pastagens das Regiões Norte e Noroeste do Estado do Rio de Janeiro. 3. Matéria Orgânica, Alumínio e pH dos Solos ${ }^{1}$
}

\author{
Fernando Luiz Henriques Tebaldi ${ }^{2}$, José Fernando Coelho da Silva ${ }^{3}$, Hernan Maldonado \\ Vasquez ${ }^{4}$, José Tarcísio Lima Thiebaut ${ }^{5}$
}

\begin{abstract}
RESUMO - Os teores de matéria orgânica e alumínio e o pH dos solos predominantes nas pastagens das regiões norte e noroeste do Estado do Rio de Janeiro foram estudados nas épocas seca (junho-julho) e chuvosa (dezembro-janeiro) do ano. Foram coletadas amostras de solo em 12 diferentes locais das regiões, em áreas onde predominam pastagens, identificadas por fotos obtidas por satélite, de acordo com as diferentes características dos solos. Os teores de Al das amostras foram determinados por espectrometria via plasma, após preparados os estratos. Os níveis de matéria orgânica dos solos não apresentaram diferenças entre épocas do ano; em todos locais estudados, foram muito baixos, sempre inferiores a 3\%. O Al não foi detectado em alguns locais e, em outros os níveis, foi muito baixo. Foi constatada correlação negativa $(\mathrm{r}=-0,85, \mathrm{P}=0,0002)$ entre níveis de $\mathrm{Al}$ e $\mathrm{pH}$ dos solos. Exceto em dois locais, os solos foram considerados ácidos, tendo o pH variado em função dos locais, sem efeito da época do ano.
\end{abstract}

Palavras chave: mineral, pastagem, $\mathrm{pH}$, solo

\section{Mineral Composition of Pastures in the North and Northwest Regions of Rio de Janeiro State. 3. Organic Matter, Aluminum and pH of the Soil}

\begin{abstract}
The levels of organic matter and aluminum and the $\mathrm{pH}$ of the predominant soils in the North and Northwest regions of Rio de Janeiro State were studied during the dry (June-July) and rainy (December-January) seasons of the year. Samples of soil were collected from 12 different places of the regions, in predominant grazing areas, as determined by satellite photography and according to different soil characteristics. The aluminum contents of the sample extracts were determined by spectrometry, on an inductive plasma emission source apparatus. Organic matter levels in the soils samples were not affected by season of year; presented very low in all sampling places and always below 3\%. Aluminum was not detected in some places and in other places the levels were very low. Negative correlation was found among aluminum levels and soil $\mathrm{pH}(\mathrm{r}=-0.85, \mathrm{P}=0.0002)$. Except for two places, the soils were considered acids with the $\mathrm{pH}$ changing in function of places, without season effect.
\end{abstract}

Key Words: mineral, pasture, $\mathrm{pH}$, soil

\section{Introdução}

A matéria orgânica do solo provém dos organismos vegetais e animais existentes no solo. Sob condições naturais, o tecido vegetal é a fonte principal de matéria orgânica nos solos. Restos de copas e raízes de árvores e arbustos, de plantas cultivadas e nativas, são anualmente incorporados ao processo de formação orgânica do solo. Dessa forma, o teor de matéria orgânica dos solos é um parâmetro que guarda certo equilíbrio, não variando drasticamente de um ano para outro. Na realidade, é um sistema complexo no qual são encontrados resíduos de plantas e animais nos mais diferentes estádios de decomposição, pro- dutos de síntese e produtos excretados pelos organismos vivos. O material não-decomposto ou parcialmente decomposto de dimensão não-coloidal é denominado de matéria orgânica livre. O material coloidal de coloração cinza-escura a preta é denominado matéria orgânica humificada ou humus, sendo essa fração humificada a responsável pelos efeitos diretos sobre as propriedades dos solos (ALMEIDA et al., 1988).

Segundo GUIMARÃES et al. (1980), quando o solo contém até $1,5 \%$ de matéria orgânica, é classificado como nível baixo; de 1,6 a 3,0\%, nível médio; e acima de 3,0\%, alto nível.

Segundo ALMEIDA et al. (1988), o teor de carbono da fração humificada do solo está relaciona-

\footnotetext{
${ }^{1}$ Parte da Dissertação de Mestrado apresentada pelo primeiro autor, à Universidade Estadual do Norte Fluminense, Campos dos Goytacazes, RJ. 2 Zootecnista, UFV, MS em Produção Animal, CCTA, UENF.

3 Docente LZNA, CCTA, UENF, Bolsista do CNPq. E.mail: jcoelho@uenf.br

${ }^{4}$ Docente LZNA, CCTA, UENF, 28015-620 Campos dos Goytacazes, RJ. E.mail: hernan@uenf.br

${ }^{5}$ Laboratório de Estatística Experimental e Análise Econômica, CCTA, UENF, 28015-620, Campos dos Goytacazes, RJ.
} 
do com aquele existente nos tecidos vegetais, animais e microbianos, que contribuíram para a sua formação. Quando resíduos vegetais ou animais são incorporados ao solo, as suas transformações e, com destaque, a mineralização são governadas, entre outros fatores, pelo teor de $\mathrm{N}$ encontrado no material. De maneira geral, essa relação $\mathrm{C} / \mathrm{N}$ situa-se em torno de 10:1 na fração humificada dos solos.

A adição de diferentes tipos de matéria orgânica ao solo produz grande modificação na sua estrutura físico-química, que, por sua vez, modifica as condições nutricionais para os microrganismos e plantas. No estado não-oxidado, a matéria orgânica fornece prontamente energia e nutrientes para o desenvolvimento microbiano do solo, e o aumento dessa atividade bioquímica resulta, por sua vez, na liberação de nutrientes essenciais para as plantas, como N, P, K, $\mathrm{S}, \mathrm{Ca}, \mathrm{Fe}, \mathrm{Mn}$ e outros (PEREIRA e PERES, 1985).

O solo é um dos componentes de um ecossistema agrícola que pode ser modificado mais facilmente pelo homem. Nos trópicos úmidos, há predominância de solos ácidos, e como a correção da acidez dos mesmos é feita por intermédio da calagem, essa é considerada necessária para se obter boa produtividade das forrageiras. Nos trópicos, há predominância de solos de carga variável, latossolos e solos podsólicos, que são normalmente ácidos. A acidificação do solo pode ser visualizada como um processo contínuo, onde diversas fontes contribuem para a mesma: 1 - hidrogênio resultante da hidrólise do $\mathrm{CO}_{2}$ produzido pela biomassa; 2 - dissociação de hidrogênio dos radicais orgânicos; e 3 - hidrogênio resultante do metabolismo vegetal excretado diretamente na rizosfera. Quando a atividade de hidrogênio na solução se torna elevada $(\mathrm{pH}<6)$, as argilas silicatadas são solubilizadas e o alumínio, liberado para a solução dos solos (SIQUEIRA, 1985).

Segundo GUIMARÃES et al. (1980), os solos com pH abaixo de 5, entre 5 e 6 e acima de 6 são classificados como ácido, médio e alto, respectivamente.

MC DOWELL et al. (1983) relata que há muita lixiviação nos solos das regiões tropicais sujeitas a fortes chuvas e altas temperaturas, tornando-os deficientes em minerais para as plantas. Quando a acidez do solo aumenta, a disponibilidade e a absorção pelas plantas de $\mathrm{Fe}, \mathrm{Mn}, \mathrm{Zn}, \mathrm{Cu}$ e Co decrescem, enquanto as concentrações de matéria orgânica (MO) e Se aumentam.

A concentração de $\mathrm{H}^{+}$não afeta diretamente o desenvolvimento vegetal, exceto em casos extremos, contudo, as condições resultantes de $\mathrm{pH}$ mais baixo são limitantes ao desenvolvimento das plantas, tanto pelo efeito sobre a disponibilidade de nutrientes, como pela concentração de Al em níveis tóxicos para a planta. A toxidez vegetal causada pelo Al solúvel é particularmente severa a valores de $\mathrm{pH}$ abaixo de 5 , em que a solubilidade de Al aumenta abruptamente (Magistad, em 1925, citado por SIQUEIRA, 1985). O Al na solução do solo aumenta com o decréscimo do conteúdo de matéria orgânica. Pela formação de complexo organo-Al, a matéria orgânica controla a atividade do Al na solução e também o Al trocável. O Al complexado na forma organo-mineral perde seu caráter tóxico para os vegetais (Bartlett et al., 1972, e Hargrave et al., 1981, citados por SIQUEIRA, 1985).

No presente estudo, foram estudados os teores de matéria orgânica e alumínio e o pH dos solos das regiões Norte e Noroeste do Estado do Rio de Janeiro.

\section{Material e Métodos}

A identificação dos locais, a coleta e o preparo das amostras foram descritos por TEBALDI et al. (2000).

O preparo das amostras de solos foi feito nos Laboratórios de Solos da Universidade Estadual Norte Fluminense - UENF e da Fundação Norte Fluminense de Desenvolvimento Regional (FUNDENOR).

Para o preparo das amostras de solos, utilizou-se o método Melic e, após descanso de uma noite, pipetaram-se $50 \mathrm{~mL}$ da solução sobrenadante de cada amostra. Nas amostras de solo, foram determinados os teores $\mathrm{Al}$ e matéria orgânica e o pH. O Al foi determinado por leitura direta, via plasma no Laboratório da ARACRUZ Celulose S.A., no aparelho ICAP 61 E PURGE (Inductively Coupled Plasma Emission Source), Thermo Jarrell Ash, por rádio frequência a $600^{\circ} \mathrm{K}$, com Argônio, para estabilizar a nebulização das amostras.

O teor de matéria orgânica foi determinado no laboratório de solos da FUNDENOR, utilizando-se $0,5 \mathrm{~g}$ de solo para $10 \mathrm{~mL}$ de bicromato de potássio a $0,4 \mathrm{~N}$, para os solos com até $2 \%$ de carbono; para maiores teores de carbono, utilizou-se $0,5 \mathrm{~g}$ de solo e $20 \mathrm{~mL}$ de bicromato de potássio - faixa em que se conseguiu a viragem para a cor alaranjado (BLOISE et al., 1979).

$\mathrm{O}$ pH das amostras de solos também foi determinado no Laboratório da FUNDENOR, em água, em potenciômetro na relação solo:água, $10 \mathrm{~g}: 25 \mathrm{~mL}$, conforme BLOISE et al. (1979).

Os demais procedimentos e interpretações dos resultados foram descritos por TEBALDI et al. (2000). 


\section{Resultados e Discussão}

\section{Matéria orgânica}

Não houve diferença nos teores de MO dos solos (Tabela 1) entre épocas, constatando-se diferença entre locais $(\mathrm{P}<0,01)$ e profundidades $(\mathrm{P}<0,05)$. Desdobrando-se a interação de época dentro de local e profundidade, constatou-se diferença nos locais 1 , 2 e 12 , na profundidade de $20 \mathrm{~cm}$, e no local 6 , nas profundidades de 20 e $40 \mathrm{~cm}(\mathrm{P}<0,05)$.

De acordo com a classificação de GUIMARÃES et al. (1980), pode-se afirmar que nenhum dos locais estudados neste trabalho atingiu a classificação de alto teor de MO, já que todos apresentaram menos de $3,00 \%$ de $\mathrm{MO}$ em seus solos. Ainda de acordo com os mesmos pesquisadores, pode-se concluir que os locais $12,6,7$ e 5 se enquadram na categoria de solos com médio teor de MO e os locais 11, 2, 3, 8, 1, 9, 10 e 4, como solos de baixo nível de MO.

Analisando os valores extremos do teor de MO, constatou-se que os resultados foram normais, pois o teor médio das duas profundidades de 0,99\% de MO, encontrado em S.J.Barra, refere-se ao solo de areia quartzoza distrófico (Restinga) e o teor médio das duas profundidades de 2,68\% de MO, encontrado em Campos-Itereré, a um solo tipo Gley pouco húmico distrófico textura média/argila (Várzea).

\section{Alumínio e $\mathrm{pH}$}

Os teores de $\mathrm{Al}$ nos solos (Tabela 2) foram extremamente baixos, não tendo sido interpretados estatisticamente. Observaram-se seis locais onde o teor de Al foi muito baixo ou não foi detectada sua presença $(8$, 10, 11, 2, 6 e 1). Os locais 10, 11, 2 e 6 referem-se a regiões de jazida calcária ou estão bem próximos dela; no caso dos locais 8 e 1, por ocasião da formação das pastagens, foi feita aplicação de calcário nos solos.

Foi encontrado coeficiente de correlação negativa entre níveis de $\mathrm{Al}$ e o pH dos solos $(\mathrm{r}=-0,85, \mathrm{P}=0,0002)$.

Os níveis de $\mathrm{pH}$ nos solos variaram $(\mathrm{P}<0,01)$ em

Tabela 1 - Teores médios de MO (\%) nos solos, nas épocas seca (Ep1) e chuvosa (Ep2), nas profundidades de $20 \mathrm{~cm}$ (1) e $40 \mathrm{~cm}$ (2) nos diferentes locais e seus respectivos números de identificação $(\mathrm{N})$

Table 1 - Average organic matter contents $(\% g)$ of soils, in the dry (Ep1) and rainy (Ep2) seasons, in the depths of 20 (1) and 40 (2), in the different places, and the respective identification numbers $(N)$

\begin{tabular}{|c|c|c|c|c|c|}
\hline $\begin{array}{l}\text { Local } \\
\text { Place }\end{array}$ & $\mathrm{N}$ & $\begin{array}{c}\text { Profundidade } \\
\text { Depth }\end{array}$ & Ep1 & Ep2 & $\begin{array}{l}\text { Média } \\
\text { Mean }\end{array}$ \\
\hline \multirow[t]{2}{*}{ Campos-Itereré } & 12 & 1 & $1,13^{\mathrm{a}}$ & $5,20^{\mathrm{b}}$ & 3,17 \\
\hline & & 2 & $2,63^{a}$ & $1,77^{\mathrm{a}}$ & 2,20 \\
\hline \multirow[t]{2}{*}{ Miracema } & 7 & 1 & $1,30^{\mathrm{a}}$ & $1,83^{\mathrm{a}}$ & 1,57 \\
\hline & & 2 & $3,53^{\mathrm{a}}$ & $1,60^{\mathrm{a}}$ & 2,57 \\
\hline \multirow[t]{2}{*}{ Italva } & 11 & 1 & $1,20^{\mathrm{a}}$ & $2,13^{\mathrm{a}}$ & 1,67 \\
\hline & & 2 & $1,57^{\mathrm{a}}$ & $1,27^{\mathrm{a}}$ & 1,42 \\
\hline \multirow[t]{2}{*}{ S.M.Madalena } & 3 & 1 & $1,37^{\mathrm{a}}$ & $1,70^{\mathrm{a}}$ & 1,53 \\
\hline & & 2 & $1,37^{\mathrm{a}}$ & $1,20^{\mathrm{a}}$ & 1,28 \\
\hline \multirow[t]{2}{*}{ Campos-Caxeta } & 9 & 1 & $0,90^{\mathrm{a}}$ & $1,43^{\mathrm{a}}$ & 1,17 \\
\hline & & 2 & $1,23^{\mathrm{a}}$ & $1,00^{\mathrm{a}}$ & 1,12 \\
\hline \multirow{2}{*}{ Itaperuna } & 10 & 1 & $1,47^{\mathrm{a}}$ & $1,03^{\mathrm{a}}$ & 1,25 \\
\hline & & 2 & $0,90^{\mathrm{a}}$ & $1,00^{\mathrm{a}}$ & 0,95 \\
\hline \multirow[t]{2}{*}{ Campos-Carvão } & 5 & 1 & $1,53^{\mathrm{a}}$ & $2,50^{\mathrm{a}}$ & 2,02 \\
\hline & & 2 & $0,97^{\mathrm{a}}$ & $1,47^{\mathrm{a}}$ & 1,22 \\
\hline \multirow[t]{2}{*}{ Campos-S.Maria } & 2 & 1 & $1,10^{\mathrm{a}}$ & $1,97^{\mathrm{b}}$ & 1,53 \\
\hline & & 2 & $0,93^{\mathrm{a}}$ & $1,63^{\mathrm{a}}$ & 1,28 \\
\hline \multirow[t]{2}{*}{ S.A.Pádua } & 8 & 1 & $0,72^{\mathrm{a}}$ & $1,60^{\mathrm{a}}$ & 1,16 \\
\hline & & 2 & $1,07^{\mathrm{a}}$ & $1,77^{\mathrm{b}}$ & 1,42 \\
\hline \multirow[t]{2}{*}{ S.F.Itabapoana } & 1 & 1 & $0,97^{\mathrm{a}}$ & $1,30^{\mathrm{b}}$ & 1,13 \\
\hline & & 2 & $1,20^{\mathrm{a}}$ & $1,27^{\mathrm{a}}$ & 1,23 \\
\hline \multirow{2}{*}{ B.J.Itabapoana } & 6 & 1 & $4,30^{\mathrm{a}}$ & $1,40^{\mathrm{a}}$ & 2,85 \\
\hline & & 2 & $1,77^{b}$ & $0,87^{\mathrm{a}}$ & 1,32 \\
\hline \multirow[t]{2}{*}{ S.J.Barra } & 4 & 1 & $1,17^{\mathrm{a}}$ & $0,50^{\mathrm{a}}$ & 0,83 \\
\hline & & 2 & $1,83^{\mathrm{a}}$ & $0,43^{\mathrm{a}}$ & 1,13 \\
\hline
\end{tabular}

Dentro de cada local e para cada profundidade, médias seguidas de mesma letra não diferem, pelo teste $\mathrm{F}$, a $5 \%$ de probabilidade.

Within each place and foreach depth, meansfollowed by the sameletterdo notdiffer, by Ftest, at $5 \%$ level of probability. 
TEBALDI et al.

função dos locais e das épocas $(\mathrm{P}<0,01)$ estudadas, mas não diferiram $(\mathrm{P}>0,05)$ quanto à profundidade, apresentando médias de 5,41 e 5,42 para as profundidades de 20 e $40 \mathrm{~cm}$, respectivamente (Tabela 3 ).

Tabela 2 - Teores médios de Al (meq/100g) nos solos, nas épocas seca (Ep1) e chuvosa (Ep2), nas profundidades de 20 (P1) e $40 \mathrm{~cm}$ (P2), nos diferentes locais, e seus respectivos números de identificação $(\mathrm{N})$

Table 2 - Average Al contents (meq/ $\mathrm{kg}$ ) of soils, in the dry (Ep1) and rainy (Ep2) seasons, in the depths of 20 (P1) and $40 \mathrm{~cm}(P 2)$, in the different places, and the respective identification numbers $(N)$

\begin{tabular}{lcccccc}
\hline $\begin{array}{l}\text { Local } \\
\text { Place }\end{array}$ & $\mathrm{N}$ & $\mathrm{Ep1}$ & $\mathrm{Ep} 2$ & $\mathrm{P} 1$ & $\mathrm{P} 2$ & $\begin{array}{c}\text { Média } \\
\text { Mean }\end{array}$ \\
\hline Miracema & 7 & 0,55 & 0,88 & 0,48 & 0,05 & 0,72 \\
B.J.Itabapoana & 6 & 0,02 & 0,10 & 0,07 & 0,05 & 0,06 \\
S.M.Madalena & 3 & 1,13 & 1,12 & 1,12 & 1,13 & 1,12 \\
S.J.Barra & 4 & 0,20 & 0,22 & 0,23 & 0,18 & 0,21 \\
S.F.Itabapoana & 1 & 0,16 & $\mathrm{ND}$ & 0,08 & 0,08 & 0,08 \\
Campos-S.Maria & 2 & 0,07 & $\mathrm{ND}$ & 0,03 & 0,03 & 0,03 \\
Italva & 11 & $\mathrm{ND}$ & 0,03 & $\mathrm{ND}$ & 0,03 & 0,02 \\
Itaperuna & 10 & $\mathrm{ND}$ & $\mathrm{ND}$ & $\mathrm{ND}$ & $\mathrm{ND}$ & $\mathrm{ND}$ \\
S.A.Pádua & 8 & $\mathrm{ND}$ & $\mathrm{ND}$ & $\mathrm{ND}$ & $\mathrm{ND}$ & $\mathrm{ND}$ \\
Campos-Carvão & 5 & 3,08 & 1,63 & 2,40 & 2,32 & 2,36 \\
Campos-Itereré & 12 & 2,80 & 0,90 & 1,77 & 1,93 & 1,85 \\
Campos-Caxeta & 9 & 1,00 & 0,90 & 0,88 & 1,02 & 0,95 \\
\hline
\end{tabular}

$\mathrm{ND}=$ Não-detectado.

$N D=$ No detectable.

Tabela 3 - pH médio dos solos, nas épocas seca (Ep1) e chuvosa (Ep2), nas profundidades de 20 (1) e $40 \mathrm{~cm}$ (2), nos diferentes locais, e seus respectivos números de identificação $(\mathrm{N})$

Table 3 - Average $\mathrm{pH}$ values of soils, in the dry (Ep1) and rainy (Ep2) seasons, in the depths of 20 (1) and $40 \mathrm{~cm}$ (2), in the different places, and the respective identification numbers $(N)$

\begin{tabular}{|c|c|c|c|c|c|}
\hline $\begin{array}{l}\text { Local } \\
\text { Place }\end{array}$ & $\mathrm{N}$ & $\begin{array}{c}\text { Profundidade } \\
\text { Depth }\end{array}$ & Ep 1 & Ep 2 & $\begin{array}{l}\text { Média } \\
\text { Mean }\end{array}$ \\
\hline \multirow[t]{2}{*}{ Itaperuna } & 10 & 1 & $7,67^{\mathrm{a}}$ & $7,33^{\mathrm{a}}$ & 7,50 \\
\hline & & 2 & $7,00^{\mathrm{a}}$ & $7,33^{\mathrm{a}}$ & 7,17 \\
\hline \multirow[t]{2}{*}{ Italva } & 11 & 1 & $6,00^{\mathrm{a}}$ & $6,00^{\mathrm{a}}$ & 6,00 \\
\hline & & 2 & $6,00^{\mathrm{a}}$ & $6,33^{\mathrm{a}}$ & 6,17 \\
\hline \multirow[t]{2}{*}{ Campos-S.Maria } & 2 & 1 & $6,00^{\mathrm{a}}$ & $6,33^{\mathrm{a}}$ & 6,17 \\
\hline & & 2 & $6,33^{\mathrm{a}}$ & $5,67^{\mathrm{a}}$ & 6,00 \\
\hline \multirow[t]{2}{*}{ S.F.Itabapoana } & 1 & 1 & $6,00^{\mathrm{a}}$ & $6,33^{\mathrm{a}}$ & 6,17 \\
\hline & & 2 & $5,67^{\mathrm{a}}$ & $6,00^{\mathrm{a}}$ & 5,83 \\
\hline \multirow[t]{2}{*}{ B.J.Itabapoana } & 6 & 1 & $5,67^{\mathrm{a}}$ & $6,00^{\mathrm{a}}$ & 5,83 \\
\hline & & 2 & $6,00^{\mathrm{a}}$ & $6,33^{\mathrm{a}}$ & 6,17 \\
\hline \multirow[t]{2}{*}{ S.A.Pádua } & 8 & 1 & $5,67^{\mathrm{a}}$ & $6,00^{\mathrm{a}}$ & 5,83 \\
\hline & & 2 & $5,67^{\mathrm{a}}$ & $6,00^{\mathrm{a}}$ & 5,83 \\
\hline \multirow[t]{2}{*}{ S.J.Barra } & 4 & 1 & $5,00^{\mathrm{a}}$ & $5,00^{\mathrm{a}}$ & 5,00 \\
\hline & & 2 & $5,33^{\mathrm{a}}$ & $6,33^{\mathrm{a}}$ & 5,83 \\
\hline \multirow[t]{2}{*}{ Campos-Caxeta } & 9 & 1 & $5,00^{\mathrm{a}}$ & $5,00^{\mathrm{a}}$ & 5,00 \\
\hline & & 2 & $5,00^{\mathrm{a}}$ & $5,67^{\mathrm{a}}$ & 5,33 \\
\hline \multirow[t]{2}{*}{ Miracema } & 7 & 1 & $(5,07)$ & $(5,00)$ & $(5,03)$ \\
\hline & & 2 & $(4,67)$ & $(4,83)$ & $(4,75)$ \\
\hline \multirow{2}{*}{ S.M.Madalena } & 3 & 1 & $(4,77)$ & $(4,90)$ & $(4,83)$ \\
\hline & & 2 & $(4,77)$ & $(4,70)$ & $(4,73)$ \\
\hline \multirow[t]{2}{*}{ Campos-Itereré } & 12 & 1 & $4,00^{a}$ & $4,67^{a}$ & 4,33 \\
\hline & & 2 & $4,00^{\mathrm{a}}$ & $5,00^{\mathrm{a}}$ & 4,50 \\
\hline \multirow{2}{*}{ Campos-Carvão } & 5 & 1 & $4,00^{\mathrm{a}}$ & $4,67^{\mathrm{a}}$ & 4,33 \\
\hline & & 2 & $4,00^{\mathrm{a}}$ & $4,67^{\mathrm{a}}$ & 4,33 \\
\hline
\end{tabular}

Dentro de cada local e para cada profundidade, médias seguidas de mesma letra não diferem entre si, pelo teste de F, em nível de $5 \%$ de probabilidade (Within each place and for each depth, means followed by the same letter do not differ, by $F$ test, at $5 \%$ level of probability).

( ) Média sem variação (Means without variation). 
386 Rev. bras. zootec.

Todavia, com desdobramento de época dentro de local e profundidade, não foi observado efeito $(\mathrm{P}>0,05)$ de época. Considerando o $\mathrm{pH}$ médio das duas profundidades, o valor mais alto de 7,21 foi observado em Itaperuna, enquanto o mais baixo, 4,18, em Campos-Carvão. Os valores médios de $\mathrm{pH}$ entre épocas estão bem próximos, para época seca e chuvosa, 5,32 e 5,51, respectivamente.

Conforme GUIMARÃES et al. (1980), os solos dos locais $9,7,3,12$ e 5 são classificados como de baixo $\mathrm{pH}$; os locais 2, 1, 6, 8 e 4, médio pH; e os municípios de Itaperuna e Italva, pH alto, já que seus valores são superiores a 6,0; esses dois últimos municípios abrangem área de uma fonte de extração de calcário.

\section{Conclusões}

Os solos de nenhum dos locais estudados apresentaram alto teor de MO.

Trata-se de uma região de solos ácidos, pois somente dois dos locais estudados apresentaram valores de $\mathrm{pH}$ elevados.

O alumínio não foi detectado em alguns locais e, em outros, os níveis foram muito baixos.

\section{Agradecimento}

Ao Prof. Dr. Doracy Pessoa Ramos do Laboratório de Solos, do Centro de Ciências e Tecnologias Agropecuárias da UENF, pela liberação das fotos da região, e ao Dr. Celso Vainer Manzatto, pela identificação e classificação dos solos.

À ARACRUZ CELULOSE S.A., na pessoa do Dr. Sebastião da Fonseca, pelas facilidades oferecidas nas análises das amostras.

\section{Referências Bibliográficas}

ALMEIDA, D.L., SANTOS, G.A., DE POLLI, H. et al. 1988. Manual de adubação para o Estado do Rio de Janeiro. $\mathrm{n}^{\circ} 2$ Editora Universidade Rural. Itaguaí, RJ. 178p.

BLOISE, R.M., MOREIA, G.N.C., DYNIA, J.F. 1979. Métodos de análises de solos e calcáreos. EMBRAPA. Serviço Nacional de Levantamento e Conservação de Solos. Boletim Técnico $\mathrm{n}^{\circ} 55$. Rio de Janeiro.

GUIMARÃES, P.T.G., FERREIRA, J.G., CARVALHO, J.G. et al. 1980. Adubação de pastagens. Informe Agropecuário, 6(70):34-52.

McDOWELL, L.R., CONRAD, J.H., ELLIS, G.L., LOOSLI, J.K. 1983. Minerals for grazing ruminant in tropical regions. The U.S Agency for International Development, Gainesville, Fl. 86p.

PEREIRA, J., PERES, J.R.R. 1985. Manejo da matéria orgânica. In: Solos dos Cerrados: Tecnologias e estratégias de manejo. GOEDERT, W.J. (Ed.). São Paulo: Nobel. 422p.

SIQUEIRA, C. Calagem para plantas forrageiras. In: SIMPÓSIO SOBRE CALAGEM E ADUBAÇÃO DE PASTAGENS, 1, Nova Odessa, SP, 1985. Anais...Nova Odessa, 1985, 476p.

TEBALDI, F.L.H., COELHO DA SILVA, J.F., VASQUEZ, H.M. et al. 2000. Composição mineral das pastagens das regiões norte e noroeste do Estado do Rio de Janeiro. 1 Cálcio, fósforo, magnésio, potássio, sódio e enxofre. Rev. bras. zootec., 29(2):603-615.
Recebido em: 30/03/98

Aceito em: 27/09/99 